Ferrata Storti Foundation

\title{
Pediatric-inspired chemotherapy incorporating pegaspargase is safe and results in high rates of minimal residual disease negativity in adults up to the age of 60 years with Philadelphia chromosome-negative acute lymphoblastic leukemia
}

Haematologica 2021

Volume 106(8):2086-2094

\section{Correspondence:}

JAE H. PARK

parkj6@mskcc.org

Received: March 232020.

Accepted: September 25, 2020.

Pre-published: October 13, 2020.

https://doi.org/10.3324/haematol.2020.251686

(C)2021 Ferrata Storti Foundation

Material published in Haematologica is covered by copyright. All rights are reserved to the Ferrata Storti Foundation. Use of published material is allowed under the following terms and conditions:

https://creativecommons.org/licenses/by-nc/4.0/legalcode. Copies of published material are allowed for personal or internal use. Sharing published material for non-commercial purposes is subject to the following conditions:

https://creativecommons.org/licenses/by-nc/4.0/legalcode, sect. 3. Reproducing and sharing published material for commercial purposes is not allowed without permission in writing from the publisher.
Mark B. Geyer, ${ }^{1,2}$ Ellen K. Ritchie, ${ }^{3}$ Arati V. Rao, ${ }^{4}$ Shreya Vemuri, ${ }^{5}$ Jessica Flynn, ${ }^{6}$ Meier Hsu6, Sean M. Devlin, ${ }^{6}$ Mikhail Roshal, ${ }^{7}$ Qi Gao, ${ }^{7}$ Madhulika Shukla, ${ }^{1}$ Jose M. Salcedo, ${ }^{1}$ Peter Maslak, ${ }^{1}$ Martin S. Tallman, ${ }^{1}$ Dan Douer ${ }^{1}$ and Jae H. Park ${ }^{1,2}$

${ }^{1}$ Leukemia Service, Department of Medicine, Memorial Sloan Kettering Cancer Center, New York, NY; ${ }^{2}$ Center for Cell Engineering, Department of Medicine, Memorial Sloan Kettering Cancer Center, New York, NY; ${ }^{3}$ Weill Cornell Medical College, Hematology and Medical Oncology, Joan and Sanford I. Weill Department of Medicine, New York, NY; ${ }^{4}$ Kite-A Gilead Company, Foster City, CA; ${ }^{5}$ Sloan Kettering Institute, New York, NY; ${ }^{6}$ Department of Epidemiology and Biostatistics, Memorial Sloan Kettering Cancer Center, New York, NY and ${ }^{7}$ Department of Pathology, Memorial Sloan Kettering Cancer Center, New York, NY, USA

\section{ABSTRACT}

dministration of pediatric-inspired chemotherapy to adults up to age 60 with acute lymphoblastic leukemia (ALL) is challenging in part due to toxicities of asparaginase as well as myelosuppression. We conducted a multi-center phase II clinical trial (clinicaltrials gov. Identifier: NCT01920737) investigating a pediatric-inspired regimen, based on the augmented arm of the Children's Cancer Group 1882 proto$\mathrm{col}$, incorporating six doses of pegaspargase $2,000 \mathrm{IU} / \mathrm{m}^{2}$, rationally synchronized to avoid overlapping toxicity with other agents. We treated 39 adults aged 20-60 years (median age 38 years) with newly-diagnosed ALL $(n=31)$ or lymphoblastic lymphoma $(n=8)$. Grade 3-4 hyperbilirubinemia occurred frequently and at higher rates in patients aged 40-60 years $(n=18)$ versus $18-39$ years $(\mathrm{n}=21)$ ( $44 \%$ vs. $10 \%, P=0.025)$. However, eight of nine patients rechallenged with pegaspargase did not experience recurrent grade 3-4 hyperbilirubinemia. Grade 3-4 hypertriglyceridemia and hypofibrinogenemia were common (each 59\%). Asparaginase activity at 7 days post-infusion reflected levels associated with adequate asparagine depletion, even among those with antibodies to pegaspargase. Complete response (CR)/CR with incomplete hematologic recovery was observed post-induction in 38 of 39 (97\%) patients. Among patients with ALL, rates of minimal residual disease negativity by multi-parameter flow cytometry were $33 \%$ and $83 \%$ following induction phase I and phase II, respectively. Event-free and overall survival at 3 years $(67.8 \%$ and $76.4 \%)$ compare favorably to outcomes observed in other series. These results demonstrate pegaspargase can be administered in the context of intensive multi-agent chemotherapy to adults aged $\leq 60$ years with manageable toxicity. This regimen may serve as an effective backbone into which novel agents may be incorporated in future frontline studies. Trial registration: https://clinicaltrials.gov/ct2/show/NCT01920737

\section{Introduction}

Treatment of acute lymphoblastic leukemia (ALL) and lymphoblastic lymphoma (LBL) in children represents one of the greatest success stories in hematologic oncology and $>85 \%$ of pediatric patients with ALL are ultimately cured. ${ }^{1}$ 
However, outcomes in adults have been less encouraging, with historical 5 -year relative survival $<45 \%$ and 5 -year overall survival $<50 \%$ among adults under the age of $60 ., 3$ Several retrospective studies have suggested superior outcomes among adolescents and younger adults (AYA) treated on pediatric versus adult cooperative group studies ${ }^{4.6}$ We and others have consequently investigated adapting pediatric ALL regimens for use in younger adults. $^{7-12}$ One large, prospective US intergroup phase II clinical trial (Cancer and Leukemia Group B [CALGB] 10403 , in collaboration with the Eastern Cooperative Oncology Group and SWOG), treated 295 AYA aged 1739 years with a true pediatric ALL regimen and observed 3 -year event-free survival (EFS) of $59 \%$, promising compared with historical controls. ${ }^{13}$ However, successfully adapting pediatric ALL therapy for adults over the age of 40 presents additional challenges and the upper age limit for safe administration of pegaspargase is not clearly defined, due in part to increasing risk of asparaginaserelated toxicities with increasing age. ${ }^{8}$

L-asparaginase, a bacterial enzyme depleting serum asparagine, was historically a standard component of ALL therapy in children and AYA. In contrast to most healthy cells, ALL/LBL cells lack asparagine synthetase and are thus dependent on exogenous asparagine and uniquely sensitive to asparaginase..$^{14}$ Randomized studies have demonstrated a survival benefit in pediatric patients treated with asparaginase-containing regimens..$^{15,16}$ However, asparaginase is associated with a host of toxicities, in part related to the above effects on protein synthesis, including hepatotoxicity, hypertriglyceridemia, hyperglycemia, hypofibrinogenemia, and thrombosis, as well as risks of hypersensitivity. ${ }^{17}$ Pegaspargase consists of polyethylene glycol covalently bound to the enzyme and may be associated with decreased immunogenicity and rates of hypersensitivity reactions compared with native E. coli asparaginase, and has a considerably longer half-life. ${ }^{18-20}$ Pegaspargase has been successfully incorporated into frontline treatment of pediatric patients and younger adults with ALL. . $1,1,21,22$

We previously reported our experience utilizing a regimen incorporating two courses of induction chemotherapy and six doses of pegaspargase $2,000 \mathrm{IU} / \mathrm{m}^{2}$ at $\geq 4$ week intervals, sequenced to avoid overlapping hepatotoxicity with other agents in adults with newly-diagnosed ALL/LBL. ${ }^{7}$ We subsequently modified this regimen to exclude two myelosuppressive courses of consolidation and incorporated serial monitoring of minimal residual disease (also known as "measurable residual disease," $M R D)$. Additional changes herein included higher doses of methotrexate (MTX, $3.5 \mathrm{~g} / \mathrm{m}^{2}$ vs. $1 \mathrm{~g} / \mathrm{m}^{2}$ for BALL/LBL; $5 \mathrm{~g} / \mathrm{m}^{2}$ vs. $2.5 \mathrm{~g} / \mathrm{m}^{2}$ for T-ALL/LBL) to reflect institutional experience that MTX doses $\geq 3.5 \mathrm{~g} / \mathrm{m}^{2}$ are sufficient to treat lymphomatous leptomeningeal and brain involvement independent of intrathecal MTX, and as T lymphoblasts require a higher MTX dose to achieve optimal MTX/MTX-polyglutamate concentrations. ${ }^{23-25}$ Maintenance chemotherapy was extended from 2 to 3 years for all patients, as 3-year maintenance is commonly used for boys treated on pediatric protocols and adults broadly exhibit higher rates of relapse than children. ${ }^{26,27}$ Pegaspargase dose was uncapped, consistent with the prior study although capping at $3,750 \mathrm{IU} / \mathrm{m}^{2}$ was reported by others in an attempt to reduce toxicity. ${ }^{7,28}$ Herein, we present results of a phase II multi-center trial investigating this approach in adults up to the age of 60 years with newly-diagnosed Philadelphia chromosome-negative ( $\mathrm{Ph}$ negative) ALL/LBL.

\section{Methods}

\section{Clinical trial}

From August 2014 to July 2017, patients with newly-diagnosed, previously untreated $\mathrm{Ph}$-negative precursor B-cell or T-cell ALL/LBL, aged 18-60 years, were enrolled at participating centers (see the Online Supplementary Methods for eligibility criteria and study design). Forty-three patients signed informed consent; four were determined ineligible prior to beginning treatment ( $\mathrm{Ph}$-positive, $n=2$; mixed phenotype acute leukemia, $n=2$ ) and 39 patients received treatment on protocol.

The primary objective of the study was to determine rates of MRD negativity following induction phase I (Table 1). Secondary objectives including assessing rates of MRD negativity following induction phase II, rates of complete response (CR), overall survival (OS), event-free survival (EFS), disease-free survival (DFS), and pegaspargase toxicities.

Toxicities were graded using National Cancer Institute Common Terminology Criteria for Adverse Events (CTCAE) v4.03.

\section{Regimen design}

Study regimen details are summarized in Table 1. Treatment was adapted from the augmented arm of the Children's Cancer Group 1882 protocol, with substitution of pegaspargase for native E. coli asparaginase and use of high-dose MTX (HD-MTX) intensification versus escalating (Capizzi) MTX, ${ }^{27}$ as previously reported.' Pegaspargase was given after the second dose of HD-MTX in intensification I/II, and only after leucovorin rescue began. The protocol was approved by the Institutional Review Boards of all participating institutions.

Pegaspargase 2,000 IU $/ \mathrm{m}^{2}$ IV (not capped) was administered over 1-2 hours at $\geq 4$-week intervals. Hydrocortisone $100 \mathrm{mg}$ intravenous (IV) was given prior to each dose and 1-2 weeks of corticosteroids followed each dose for hypersensitivity prophylaxis (Table 1). A specific pegaspargase toxicity management guideline was adopted; asparaginase enzyme activity and anti-asparaginase antibodies were measured at pre-specified time points (see the Online Supplementary Methods and the Online Supplementary Table S1).

\section{Minimal residual disease assessment}

$\mathrm{MRD}$ was assessed centrally in bone marrow (BM) aspirate samples using multiparameter flow cytometry (FACS) with sensitivity of at least $10^{-4}$. Any unequivocal evidence of residual ALL by FACS was considered as MRD positivity, even if $<0.01 \%$ of BM mononuclear cells (see the Online Supplementary Methods; Online Supplementary Table S2; Online Supplementary Figure S1).

\section{Statistical analyses}

Incidence of grade 3-4 toxicities was compared between groups by age, sex, and BMI using Fisher's exact test. OS, EFS, and DFS were computed using the Kaplan-Meier method and compared between groups using log-rank tests. EFS was defined as time from initiation of protocol therapy until date of morphologic relapse, confirmed refractory disease, or death from any cause; patients not known to have any of these events were censored on date of last follow-up. OS was defined as time from start of protocol therapy to death from any cause, with surviving patients censored at last follow-up. Among patients achieving CR/CRi, 
DFS was defined as time from post-induction I or II disease assessment until morphologic relapse or death; patients without any of these events were censored on date of last follow-up. In order to compare OS between patients undergoing versus not undergoing allogeneic hematopoietic cell transplantation (alloHCT) in CR1, OS was measured from date of first confirmed CR. Statistical analyses were performed in R v3.5.0. A 2-sided Pvalue $<0.05$ was considered significant.

\section{Results}

\section{Patients characteristics}

Demographic and clinical characteristics of treated patients are summarized in Table 2 . Of the 39 patients, 30 $(77 \%)$ were men. Median age at the start of treatment was 38.7 years (range, 20.2-60.4 years); 18 (46\%) patients were 40-60 years old. Lineage was B cell in 27 (69\%) patients (ALL, $n=24$; LBL, $n=3$ ) and $T$ cell in 12 patients (ALL, $n=7$; LBL, $n=5)$. Three patients had central nervous system
(CNS)-2/3 cerebrospinal fluid $(n=2)$ or parenchymal brain involvement $(n=1)$ and 16 had extramedullary disease at diagnosis. Five of 12 patients with T-cell ALL/LBL demonstrated early T-precursor phenotype as previously defined. ${ }^{29}$ Cytogenetic findings associated with unfavorable risk (per classification used in the CALGB 19802 study; Online Supplementary Table S3) were observed in five patients (11q23 rearrangement, $n=2$; trisomy $8, n=2$; monosomy 7, n=1). Among 11 patients with B-ALL/LBL who underwent evaluation for fusions/re-arrangements characteristic of Philadelphia chromosome-like ("Ph-like") ALL (see the Online Supplementary Methods), only one case was identified (FIP1L1-PDGFRA fusion).

\section{Clinical responses}

Thirty-eight of 39 (97\%) patients achieved CR or CR with incomplete hematologic recovery (CRi) by the conclusion of induction II. Following induction I, 36 of 38 (95\%) of evaluable patients were in CR/CRi. Of the two patients who were not in CR/CRi, both had T-cell ALL;

Table 1. Treatment regimen.

\begin{tabular}{|c|c|c|c|c|}
\hline Regimen Block & Agent & Dose & Route & Days \\
\hline $\begin{array}{l}\text { Induction } \\
\text { Phase I }\end{array}$ & $\begin{array}{l}\text { Daunorubicin } \\
\text { Vincristine } \\
\text { Pegaspargase } \\
\text { Prednisone } \\
\text { Methotrexate } \\
\text { Hydrocortisone }\end{array}$ & $\begin{array}{c}60 \mathrm{mg} / \mathrm{m}^{2} \\
1.4 \mathrm{mg} / \mathrm{m}^{2}(\mathrm{cap} 2 \mathrm{mg}) \\
2,000 \mathrm{U} / \mathrm{m}^{2} \\
60 \mathrm{mg} / \mathrm{m}^{2} \\
12 \mathrm{mg} \\
25 \mathrm{mg}\end{array}$ & $\begin{array}{c}\text { IV push } \\
\text { IVPB/IV push } \\
\text { IVPB over 1-2h } \\
\text { PO } \\
\text { IT } \\
\text { IT }\end{array}$ & $\begin{array}{c}1,2,3 \\
1,8,15,22 \\
15 \\
1-28 \\
8,15 \\
8.15\end{array}$ \\
\hline $\begin{array}{l}\text { Induction } \\
\text { Phase II }\end{array}$ & $\begin{array}{l}\text { Cyclophosphamide } \\
\text { Cytarabine } \\
\text { Mercaptopurine } \\
\text { Vincristine } \\
\text { Pegaspargase } \\
\text { Prednisone } \\
\text { Methotrexate } \\
\text { Hydrocortisone2 }\end{array}$ & $\begin{array}{c}1 \mathrm{~g} / \mathrm{m} \\
75 \mathrm{mg} / \mathrm{m}^{2} \\
60 \mathrm{mg} / \mathrm{m}^{2} \\
1.4 \mathrm{mg} / \mathrm{m}^{2}(\text { cap } 2 \mathrm{mg}) \\
2,000 \mathrm{U} / \mathrm{m}^{2} \\
20 \mathrm{mg} \\
12 \mathrm{mg} \\
25 \mathrm{mg}\end{array}$ & $\begin{array}{c}\text { IVPB } \\
\text { IVPB } \\
\text { PO } \\
\text { IVPB/IV push } \\
\text { IVPB over } 1-2 \mathrm{~h} \\
\text { PO } \\
\text { IT } \\
\text { IT }\end{array}$ & $\begin{array}{c}1,29 \\
1-4,8-11,29-32,36-39 \\
1-14,29-42 \\
1,15,29,43 \\
15 \\
15-22 \\
1,15,29,43 \\
1,15,29,43\end{array}$ \\
\hline Intensification I & $\begin{array}{l}\text { Methotrexate } \\
\text { Leucovorin* } \\
\text { Pegaspargase } \\
\text { Prednisone }\end{array}$ & $\begin{array}{c}3.5 \mathrm{~g} / \mathrm{m}^{2} \text { (B-cell) } \\
5 \mathrm{~g} / \mathrm{m}^{2}(\mathrm{~T}-\text { cell }) \\
25 \mathrm{mg} \\
2,000 \mathrm{U} / \mathrm{m}^{2} \\
20 \mathrm{mg}\end{array}$ & $\begin{array}{l}\text { IVPB over } 3 \mathrm{~h} \\
\text { IVPB over } 3 \mathrm{~h} \\
\text { IVPB q6h } \\
\text { IVPB over } 1-2 \mathrm{~h} \\
\text { PO }\end{array}$ & $\begin{array}{c}1,15 \\
1,15 \\
\text { 24h from start of MTX } \\
16 \text { or } 17 \\
15-22\end{array}$ \\
\hline Re-induction I & $\begin{array}{l}\text { Daunorubicin } \\
\text { Vincristine } \\
\text { Pegaspargase } \\
\text { Dexamethasone } \\
\text { Methotrexate } \\
\text { Hydrocortisone } \\
\text { Cyclophosphamide } \\
\text { Cytarabine } \\
\text { Thioguanine }\end{array}$ & $\begin{array}{c}25 \mathrm{mg} / \mathrm{m}^{2} \\
1.4 \mathrm{mg} / \mathrm{m}^{2}(\mathrm{cap} 2 \mathrm{mg}) \\
2,000 \mathrm{U} / \mathrm{m}^{2} \\
10 \mathrm{mg} / \mathrm{m}^{2} \\
12 \mathrm{mg} \\
25 \mathrm{mg} \\
1 \mathrm{~g} / \mathrm{m}^{2} \\
75 \mathrm{mg} / \mathrm{m}^{2} \\
60 \mathrm{mg} / \mathrm{m}^{2}\end{array}$ & $\begin{array}{c}\text { IV push } \\
\text { IVPB/IV push } \\
\text { IVPB over 1-2h } \\
\text { PO } \\
\text { IT } \\
\text { IT } \\
\text { IVPB } \\
\text { IVPB } \\
\text { PO }\end{array}$ & $\begin{array}{c}1,8,15 \\
1,8,15,29,43 \\
15 \\
15-22,29-36 \\
1,8,29 \\
1,8,29 \\
29 \\
29-32,36-39 \\
29-42\end{array}$ \\
\hline Intensification II & Same as intensification I & & & \\
\hline Re-induction II & Same as re-induction I & & & \\
\hline $\begin{array}{l}\text { Maintenance } \\
\text { (Monthly for } 36 \text { months total) }\end{array}$ & $\begin{array}{l}\text { Prednisone } \\
\text { Vincristine } \\
\text { Methotrexate }\end{array}$ & $\begin{array}{c}60 \mathrm{mg} / \mathrm{m}^{2} \\
1.4 \mathrm{mg} / \mathrm{m}^{2}(\text { cap } 2 \mathrm{mg}) \\
10 \mathrm{mg} / \mathrm{m}^{2}\end{array}$ & $\begin{array}{l}\text { PO } \\
\text { IV } \\
\text { PO }\end{array}$ & $\begin{array}{l}\text { 1-5 (q mon, mon. 1-12; q2 mon, mon. 13-24) } \\
1 \text { (q mon, mon. 1-12; q2 mon, mon. 13-36) } \\
\text { 1,8,15,22 (held on days of IT MTX) }\end{array}$ \\
\hline & $\begin{array}{l}\text { Mercaptopurine } \\
\text { Methotrexate } \\
\text { Hydrocortisone } \\
\end{array}$ & $\begin{array}{l}60 \mathrm{mg} / \mathrm{m}^{2} \\
12 \mathrm{mg} \\
25 \mathrm{mg}\end{array}$ & $\begin{array}{l}\text { PO } \\
\text { IT } \\
\text { IT }\end{array}$ & $\begin{array}{l}\text { 1-28, daily } \\
1 \text { (q3 mon, mon. 1-12) } \\
1 \text { (q3 mon, mon. 1-12) }\end{array}$ \\
\hline
\end{tabular}

*Leucovorin rescue following the day 15 dose of high-dose methotrexate was started prior to administration of pegaspargase. IVPB: intravenous piggy back; PO: by mouth; IT: intrathecal; MTX: methotrexate, mon: month; cap: capped; q2: calender quarter 2 (April, May, and June); q3: calender quarter 3 (July, August, and Septembe). 
one continued protocol therapy and achieved CR following induction II and the other was removed from study after induction I at the discretion of the investigator to pursue alternative therapy (representing the single patient who did not achieve CR/CRi on study). One patient was not evaluable for response post-induction I due to incomplete restaging; this patient continued protocol therapy and was confirmed to be in CR following induction II. No patients died during induction I or II.

Central evaluation for $\mathrm{MRD}$ in $\mathrm{BM}$ was performed in 26

Table 2. Patient demographics and clinical characteristics.

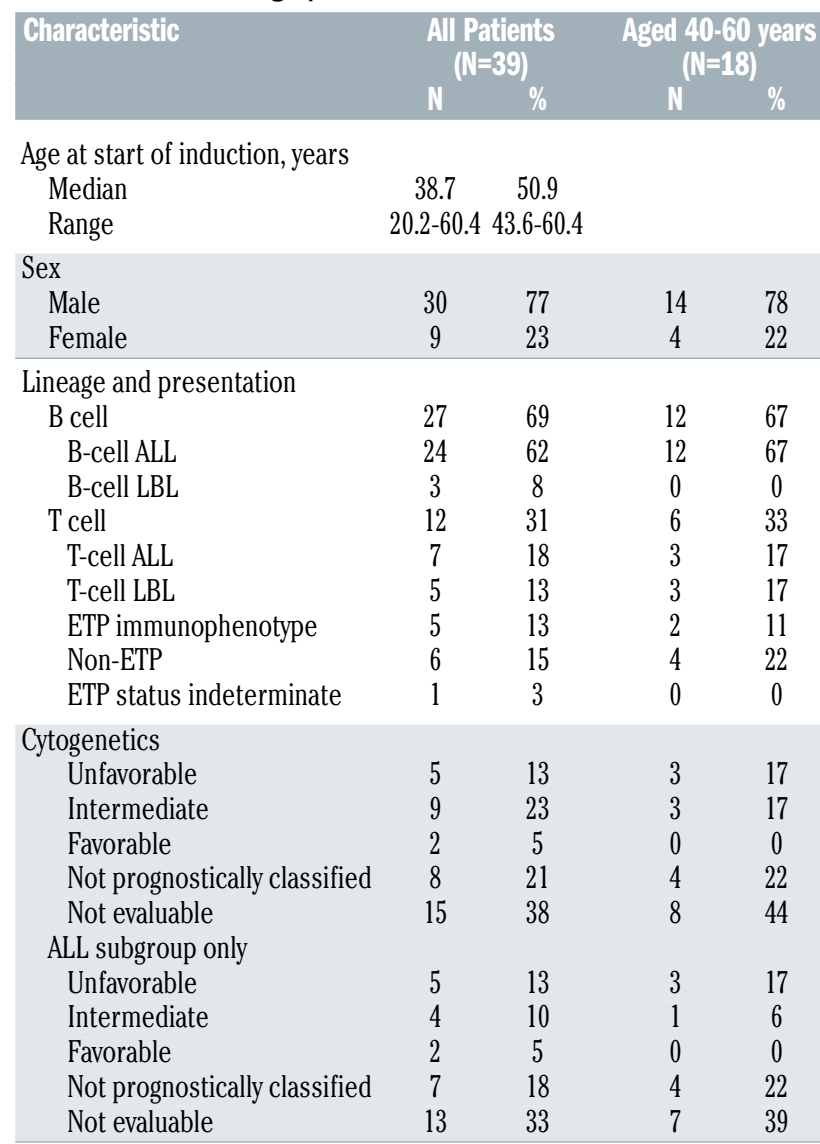

CNS disease (CNS-2/3 CSF or

parenchymal brain involvement) at diagnosis

$\begin{array}{lcccc}\text { Yes } & 3 & 8 & 0 & 0 \\ \text { No } & 36 & 92 & 18 & 100\end{array}$

Unequivocal extramedullary disease at diagnosis

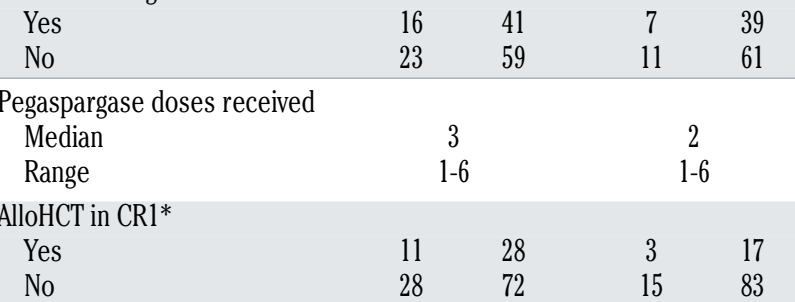

*Includes patients known to have undergone allogeneic hematopoietic cell transplantation (alloHCT) in first complete response. ALL: acute lymphoblastic leukemia; LBL: lymphoblastic lymphoma; ETP: early T-precursor phenotype (CD1a-, CD8-, CD5dim/-, with expression of one or more stem cell or myeloid antigens, e.g., CD11b, CD13, CD33, CD34, CD117, HLA-DR); cytogenetic risk group classification per criteria used in the CALGB 19802 study, are summarized in the Online Supplementary Table S3; CNS: central nervous system; CSF: cerebrospinal fluid. of 31 patients with ALL (i.e., not LBL) on day 15 of induction I; at that time, five of $26(19 \%)$ of patients had achieved MRD negativity. The proportion of patients with ALL exhibiting BM MRD negativity on central review increased following induction I (nine of 27,33\%) and induction II (20 of $24,83 \%$ ). Patients with LBL and lowlevel BM involvement at diagnosis are not included in the aforementioned analysis. However, four of four patients with low-level BM involvement by LBL exhibited MRD negativity in the BM on day 15 of induction I. Local MRD data are summarized in the Online Supplementary Results.

\section{Pegaspargase administration, enzymatic activity, and immunogenicity}

All treated patients received at least one dose of pegaspargase, and the median number of doses received was three (range, 1-6). Reasons for receipt of $<6$ total doses of pegaspargase are summarized in the Online Supplementary Table S4. Asparaginase enzymatic activity as measured 7 days post-pegaspargase is summarized in Online Supplementary Table S3. All activity levels obtained 7 days following a full dose of pegaspargase (i.e., excluding those with immediate hypersensitivity) were $>0.1 \mathrm{IU} / \mathrm{mL}$ (minimum observed level $0.34 \mathrm{IU} / \mathrm{mL}$ ). In two patients with immediate hypersensitivity to pegaspargase who received 5 and 15 minutes of the infusion, activity levels 7 days post-pegaspargase were $<0.013$ and $0.028 \mathrm{IU} / \mathrm{mL}$, respectively. Enzymatic activity appeared similar in patients ageg 40-60 years versus 18-39 years (Online Supplementary Table S5).

In 19 patients, 75 plasma samples were screened for antibodies specific to Oncaspar ${ }^{\circledR}$ and polyethylene glycol (PEG; see the Online Supplementary Methods). Inhibition with Oncaspar ${ }^{\circledast}$ showed ten confirmed positive samples and specific inhibition with $5 \mathrm{kDa}$ PEG showed six confirmed positive samples; these confirmed results were from four patients and are summarized in the Online Supplementary Table S6. Despite the presence of antibodies, asparaginase activity levels were $>0.1 \mathrm{IU} / \mathrm{mL}$ (minimum $0.34 \mathrm{IU} / \mathrm{mL}$ ) 7 days post-pegaspargase in all instances in which the full dose of pegaspargase was administered (i.e., not terminated early due to hypersensitivity).

\section{Pegaspargase toxicity}

Selected grade 3-4 toxicities of pegaspargase in all patients and in those aged 40-60 years at treatment initiation are summarized in the Online Supplementary Table S4. Grade 3-4 hypofibrinogenemia and hypertriglyc-

Table 3. Serum asparaginase enzymatic activity post-polyethylene glycol.

\begin{tabular}{lccc} 
Time point in treatment & $\begin{array}{c}\text { Samples } \\
\text { (N) }\end{array}$ & \multicolumn{2}{c}{$\begin{array}{c}\text { Serum asparaginase } \\
\text { enzymatic activity } 7 \text { days } \\
\text { post-PEG (IU/mL) } \\
\text { Mean }\end{array}$} \\
\hline Induction I & 15 & 0.787 & 0.238 \\
Induction Phase II & 14 & 0.881 & 0.234 \\
\hline Intensification I & 6 & 0.989 & 0.149 \\
Re-induction I & 7 & 0.852 & 0.238 \\
\hline Intensification II & 10 & 1.057 & 0.190 \\
Re-induction II & 7 & 1.087 & 0.145 \\
\hline All Phases & 59 & 0.919 & 0.233 \\
\hline
\end{tabular}

PEG: polyethylene glycol; SD: standard deviation. 
eridemia were common (each observed in 59\% of patients). Grade 3-4 hyperbilirubinemia was significantly more common in patients aged $40-60$ years versus $18-39$ years ( $44 \%$ vs. $10 \%, P=0.025)$; a non-significant trend toward greater risk of grade 3-4 hyperbilirubinemia was observed in patients with body mass index (BMI) $\geq 30$ versus $<30 \mathrm{~kg} / \mathrm{m}^{2}$ at treatment initiation $(50 \%$ vs. $17 \%$, $P=0.087)$. Incidence of grade 3-4 transaminitis, hypertriglyceridemia, hyperglycemia, hypofibrinogenemia, pancreatitis, and thromboembolic events otherwise did not significantly differ by age (18-39 years vs. 40-60 years), BMI ( $\geq 30 \mathrm{~kg} / \mathrm{m}^{2}$ vs. $\left.<30 \mathrm{~kg} / \mathrm{m}^{2}\right)$, or sex.

Nine of the ten patients experiencing grade 3-4 hyperbilirubinemia following pegaspargase during induction I resumed pegaspargase at the dose and schedule per protocol; eight of nine patients resuming pegaspargase did not experience recurrent grade 3-4 hyperbilirubinemia. In four patients, Erwinia asparaginase was substituted for pegaspargase due to hypersensitivity associated with pegaspargase infusion.

Other non-hematologic adverse events and febrile neutropenia definitely, probably, or possibly related to protocol therapy are detailed in the Online Supplementary Table S7. Association of pegaspargase with treatment delays is summarized in the Online Supplementary Results.

\section{Subsequent therapy and relapse}

The patient with T-ALL who was withdrawn from study after exhibiting persistent disease following induction I received second-line chemotherapy off-protocol with nelarabine, etoposide, and cyclophosphamide, and subsequently underwent alloHCT. Ten additional patients receiving protocol therapy underwent alloHCT in CR1. Rationale for alloHCT in CR1 was as follows: persistent MRD following course II of induction $(n=3)$, Ph-like ALL $(n=1)$, unfavorable cytogenetic features including $\mathrm{t}(4 ; 11)(\mathrm{n}=1)$ or evidence of clonal evolution $(n=1)$, or physician preference $(n=4)$.

Of 38 patients achieving CR/CRi on protocol, ten subsequently experienced relapse (BM, $n=4$; $C N S$ only, $n=2$; combined $\mathrm{BM}$ and $\mathrm{CNS}, \mathrm{n}=1$; combined $\mathrm{BM}$ and other extramedullary sites, $\mathrm{n}=1$; testis, $\mathrm{n}=1$; cortical bone, $\mathrm{n}=1$ ); median time to relapse from start of therapy was 15.4 months (range, 5.4-40.4) in these ten patients. Six patients underwent alloHCT following relapse, including one patient who had relapsed following first alloHCT in CR1.

\section{Survival outcomes}

Two patients died in CR1 during re-induction I due to complications of sepsis $(n=1)$ or multi-organ system failure $(n=1)$, aged 55 and 47 years, respectively, at the time of death. At median follow-up of 38.6 months among surviving patients (range, 1.8-57.8), 3-year OS is $76.4 \%$ (95\% Confidence Interval [CI]: 63.3-92.3) and 3-year EFS is $67.8 \%$ (95\% CI: 53.4-86.0), as summarized in Figure $1 \mathrm{~A}$ and $\mathrm{B}$. Three-year cumulative incidence of relapse (CIR, with death in CR as competing event) is $25.3 \%$ (95\% CI: 11.4-42.0). Superior OS and EFS were observed among patients aged 18-39 years versus 40-60 years at the start of therapy (3-year OS: 88.2 vs. $61.9 \%, P=0.03$ and 3 -year EFS: $85.0 \%$ vs. $48.6 \%, P=0.05$, respectively, Figure $1 C$ and D). OS and EFS did not differ significantly with respect to $\mathrm{B}$-cell versus $\mathrm{T}$-cell lineage (3-year OS: $78.7 \%$ vs. $71.6 \%$, $P=0.8$, and 3 -year EFS: $71.8 \%$ vs. $58.7 \%, P=0.7$, respectively). In this small study, significant differences were not observed in OS, DFS, or CIR between the 10 patients undergoing alloHCT after achieving CR1 with protocol therapy and the 27 evaluable patients not undergoing alloHCT in CR1 (3-year OS: $100 \%$ vs. $70.7 \%, P=0.3$; 3year DFS: $88.9 \%$ vs. $62.5 \%, P=0.09$; 3-year CIR: $11.1 \%$ vs. $29.8 \%, P=0.197)$.

Among the patients with ALL achieving MRD-negative CR versus MRD-positive CR/CRi (central review) following induction 1, there was no difference in OS or DFS measured from time of post-induction I disease assessment (3-year OS: $75.0 \%$ vs. $80.8 \%, P=0.8$, and 3-year DFS: $75.0 \%$ vs. $68.8 \%, P=0.5)$. Twelve patients with ALL who were in MRD-positive CR/CRi following induction I subsequently achieved $M R D$ negativity following induction II (MRD converter group) and seven patients with ALL were confirmed to be in MRD-negative CR following both induction I and induction II (early MRD negativity group). The MRD converter and early MRD negativity groups achieved 3 -year DFS $56.2 \%$ and $71.4 \%$, respectively, $P=0.7$, and 3 -year OS $90.9 \%$ and $71.4 \%$, $P=0.5$, respectively, as measured from time of post-induction II disease assessment. The small number of patients with MRD positivity post-induction II $(n=4)$ also precludes meaningful comparison of this group with those exhibiting MRD negativity post-induction II; these patients are described in the Online Supplementary Table S8. All patients with MRD positivity post-induction II underwent alloHCT (CR1, n=3; CR2, n=1) following subsequent therapy and three are in ongoing CR.

Table 4. Selected grade 3-4 toxicities possibly, probably, or definitely attributed to pegaspargase.

\begin{tabular}{|c|c|c|c|c|c|c|c|c|c|c|c|c|}
\hline \multirow[t]{3}{*}{ Toxicity } & \multicolumn{6}{|c|}{ All patients $(\mathrm{N}=39)$} & \multicolumn{6}{|c|}{ Patients aged $40-60$ years $(N=18)$} \\
\hline & \multicolumn{2}{|c|}{ Grade 3} & \multicolumn{2}{|c|}{ Grade 4} & \multicolumn{2}{|c|}{$\begin{array}{c}\text { Total } \\
\text { Grade 3-4 }\end{array}$} & \multicolumn{2}{|c|}{ Grade 3} & \multicolumn{2}{|c|}{ Grade 4} & \multicolumn{2}{|c|}{$\begin{array}{c}\text { Total } \\
\text { Grade 3-4 }\end{array}$} \\
\hline & $\mathrm{N}$ & $\%$ & $\mathrm{~N}$ & $\%$ & $\mathrm{~N}$ & $\%$ & $\mathrm{~N}$ & $\%$ & $\mathrm{~N}$ & $\%$ & $\mathrm{~N}$ & $\%$ \\
\hline Hypofibrinogenemia & 21 & 54 & 2 & 5 & 23 & 59 & 10 & 56 & 0 & 0 & 10 & 56 \\
\hline Hypertriglyceridemia & 11 & 28 & 12 & 31 & 23 & 59 & 4 & 22 & 6 & 33 & 10 & 56 \\
\hline Transaminitis & 15 & 38 & 1 & 3 & 16 & 41 & 9 & 50 & 1 & 6 & 10 & 56 \\
\hline Hyperglycemia & 8 & 21 & 4 & 10 & 12 & 31 & 4 & 22 & 2 & 11 & 6 & 33 \\
\hline Hyperbilirubinemia & 5 & 13 & 5 & 13 & 10 & 26 & 3 & 17 & 5 & 28 & 8 & 44 \\
\hline Pancreatitis & 1 & 3 & 0 & 0 & 1 & 3 & 0 & 0 & 0 & 0 & 0 & 0 \\
\hline Thromboembolic events & 1 & 3 & 0 & 0 & 1 & 3 & 1 & 6 & 0 & 0 & 1 & 6 \\
\hline
\end{tabular}

ALL: acute lymphoblastic leukemia. 


\section{Discussion}

In this phase II study, we sought to improve the toxicity profile of the regimen investigated at the University of Southern California (USC) and previously reported by members of our group, and to define rates of MRD negativity associated with this approach.' Specifically, we omitted two intensely myelosuppressive courses of consolidation (cytarabine/teniposide) and incorporated standardized post-pegaspargase laboratory monitoring to enhance the safety of this regimen, particularly for adults over the age of 40 years. We observed no deaths during induction chemotherapy and toxicities of pegaspargase were manageable, albeit common, in patients up to the age of 60 years as subsequently discussed. Removal of the cytarabine/teniposide consolidation blocks did not appear to result in inferior outcomes compared with our prior experience. ${ }^{7}$ However, two patients died during post-remission therapy, underscoring the Re-induction blocks' associated risks of myelosuppression. Incidence of febrile neutropenia (ten patients, $26 \%$ ) herein was comparable to other pediatric regimens ${ }^{10,13}$ though not reported directly with the USC regimen. Further follow-up will be needed to assess whether longer duration of maintenance chemotherapy will be associated with lower risk of late relapse. Despite incorporation of higher-dose MTX in the updated regimen and 16 doses of intrathecal MTX given throughout treatment in both regimens, three patients experienced CNS relapse (isolated or with marrow relapse). Neither this regimen nor its USC predecessor incorporated cranial radiation; further follow-up and clinical experience will clarify long-term rates of CNS relapse associated with this regimen. This study also provides further data on kinetics of MRD clearance in adults over the age of 40 treated with a pediatric-inspired regimen, whereas much of the existing MRD data utilizing similar regimens reflects treatment of children, adolescents, and younger adults. OS and EFS compare favorably to outcomes historically observed in adults with ALL/LBL.

Others have also investigated incorporation of pegaspargase into frontline treatment paradigms for adults with ALL. The UK National Cancer Research Institute UKALL14 trial evaluated pegaspargase $1,000 \mathrm{IU} / \mathrm{m}^{2}$ given on days 4 and 18 in combination with daunorubicin, vincristine, and dexamethasone, \pm rituximab (B-cell ALL) and imatinib ( $\mathrm{Ph}$-positive), in patients aged $25-65$ years with newly-diagnosed ALL. ${ }^{8}$ However, 16 of 90 patients succumbed to treatment-related mortality during induction, with 11 deaths related to hepatotoxicity \pm infection and associated with grade 3-4 hyperbilirubinemia in nine patients. However, while patients with $\mathrm{Ph}+\mathrm{ALL}$ accounted for only $29 \%$ of study participants, those with $\mathrm{Ph}+$ ALL accounted for 11 of 16 deaths during induction and
A

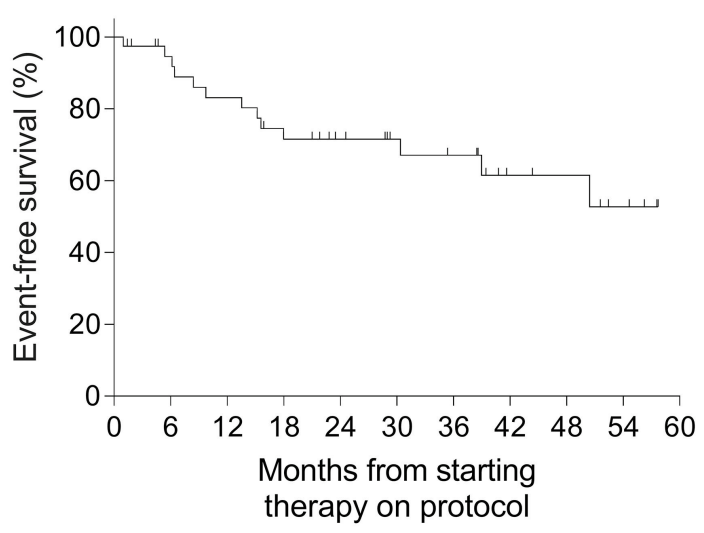

C

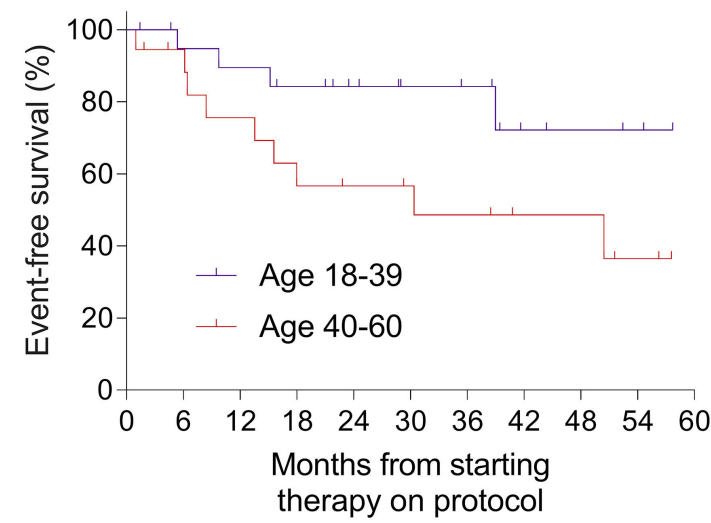

B

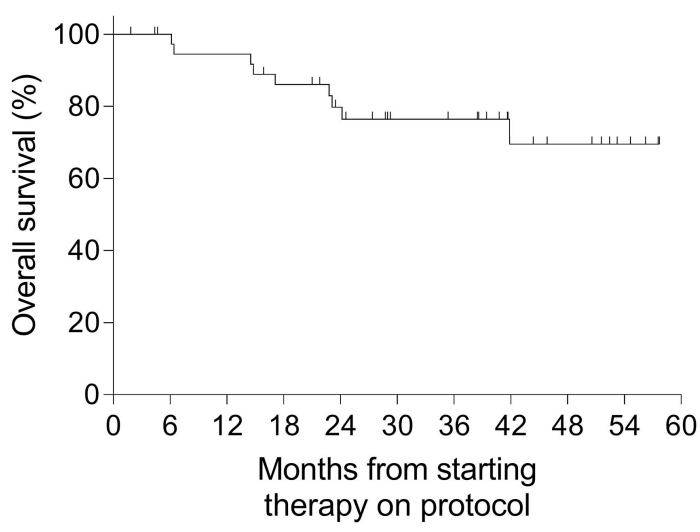

D

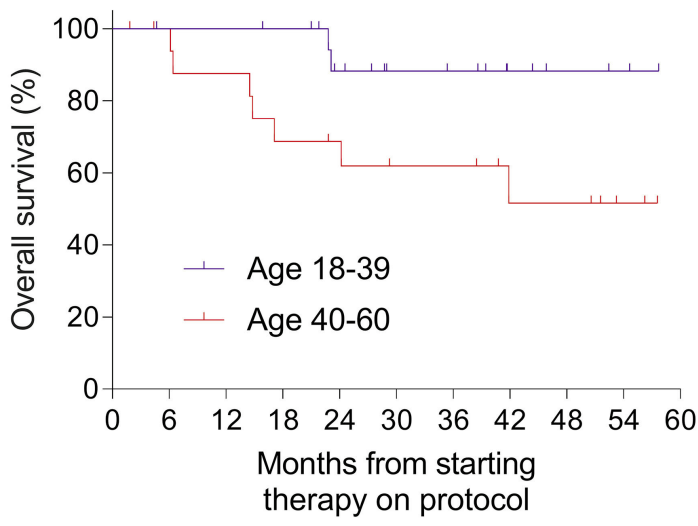

Figure 1. Survival outcomes among treated patients. (A) Event-free survival (EFS) (freedom from morphologic relapse, removal from study for refractory disease, or death) and (B) overall survival (OS) among all treated patients. (C) EFS and (D) OS among patients aged 18-39 years vs. 40-60 years at initiation of therapy ( $P=0.06$ and $P=0.03$, respectively). 
seven of 11 hepatotoxicity-associated deaths (OR 8.65, $P<0.001)$. UKALL14 data suggest the combination of imatinib and pegaspargase may have been particularly toxic, though similarly severe toxicity was not observed in patients with $\mathrm{Ph}+\mathrm{ALL}$ receiving imatinib in combination with the USC regimen.' In contrast, our study did not include patients with $\mathrm{Ph}+$ ALL. Additionally, use of pegaspargase $1,000 \mathrm{IU} / \mathrm{m}^{2}$ on days 4 and 18 of induction (vs. 2,000 IU $/ \mathrm{m}^{2}$ on day 15 in this regimen) may have resulted in different duration of asparagine depletion, and in the context of the UKALL14 regimen, weekly dosing of daunorubicin $60 \mathrm{mg} / \mathrm{m}^{2}\left(v s .60 \mathrm{mg} / \mathrm{m}^{2}\right.$ days $1-3$ in this regimen) may have led to greater overlap of hepatoxicity from pegaspargase and intense myelosuppression from the anthracycline. Similarly, patients aged $46-60$ years versus 15-45 years treated on the GRAALL-2003 study tended to have higher rates of grade 3-4 hepatotoxicity during induction $(28 \%$ vs. $18 \%)$, received significantly lower median cumulative dose of L-asparaginase during induction $\left(36,000 \mathrm{IU} / \mathrm{m}^{2}\right.$ vs. $\left.48,000 \mathrm{IU} / \mathrm{m}^{2}\right)$ and post-remission $\left(29,000 \mathrm{IU} / \mathrm{m}^{2}\right.$ vs. $\left.81,000 \mathrm{IU} / \mathrm{m}^{2}\right)$, experienced delays in consolidation initiation, and exhibited significantly increased risk of death during induction $(13 \% v s .4 \%)$ and cumulative incidence of death in first CR (15\% vs. $2 \%){ }^{9}$ Administration of cyclophosphamide and daunorubicin in proximity to L-asparaginase during induction with the GRAALL-2003 regimen may also have contributed to toxicity. Like other series, toxicities associated with pegaspargase were common in our study, and grade 3-4 hyperbilirubinemia was observed more commonly in patients aged 40-60 years versus $18-39$ years, albeit without evidence of acute liver failure or long-term hepatic morbidity. Notably, nearly all patients experiencing grade 3-4 hyperbilirubinemia were able to be re-challenged with the drug without recurrent severe toxicity, suggesting that a single episode of grade 3-4 hyperbilirubinemia does not necessitate pegaspargase discontinuation in adults with ALL, even in patients aged 40-60 years. We observed no deaths during induction chemotherapy, though two patients aged 40-60 years ultimately died in CR1 during post-remission therapy. The rate of thrombosis was low $(3 \%)$, perhaps due in part to chance as routine antithrombotic prophylaxis was not used (Online Supplementary Table S1); the USC regimen was associated with a $16 \%$ incidence of deep vein thrombosis, similar to other pediatric regimens studied in this age group., ${ }^{710}$ Our restrictive approach to fibrinogen repletion ( $<50 \mathrm{mg} / \mathrm{dL}$ or bleeding) may have limited thrombotic risk as well, consistent with other recent reports. ${ }^{30}$ This regimen may serve as an effective option for patients aged 40-60 years, particularly for patients for whom an asparaginase-containing regimen may be especially desirable (e.g. T-ALL/LBL). ${ }^{15,31,32}$

Asparaginase activity levels measured at 7 days following a full dose of pegaspargase were uniformly in the range associated with asparagine depletion $(>0.1 \mathrm{IU} / \mathrm{mL}$, with minimum observed level $0.34 \mathrm{IU} / \mathrm{mL}$ herein) across all age groups. Mean asparaginase activity at 7 days postdose was $0.601 \mathrm{IU} / \mathrm{mL}$ in our prior pharmacokinetic study of pegaspargase 2,000 IU $/ \mathrm{m}^{2}$ during remission induction for younger adults with ALL, and in that series, 12 of 12 and nine of 11 patients exhibited asparagine depletion at 14 and 20 of 21 days post-pegaspargase, respectively. ${ }^{33}$ In this study, mean asparaginase activity at 7 days was 0.919 $\mathrm{IU} / \mathrm{mL}$ (Table 3), suggesting at least similarly robust asparagine depletion, though activity levels were not directly measured at 14 and 21 days post-dose. Of note, even in patients developing antibodies to pegaspargase, there was no evidence of silent inactivation, providing some reassurance against this phenomenon as a major mechanism of therapeutic resistance in the enrolled patients, despite prophylactic administration of hydrocortisone to all patients. Absence of silent inactivation further suggests routine monitoring of asparaginase activity following each pegaspargase may not be essential. Whether pegaspargase doses $<2,000 \mathrm{IU} / \mathrm{m}^{2}$ might achieve similar enzymatic activity levels and asparagine depletion within the context of this regimen, while leading to lower rates of severe toxicity, remains unclear. However, among patients treated on the GMALL07/2003 protocol, pegaspargase doses of $1,000 \mathrm{IU} / \mathrm{m}^{2}$ or $2,000 \mathrm{IU} / \mathrm{m}^{2}$ resulted in asparaginase activity targets achieved in $96 \%$ and $98 \%$ of patients in the second week post-dose, and 73 and $89 \%$ in the third week post-dose, respectively. ${ }^{34}$

As noted previously, CALGB 10403 demonstrated the safety and efficacy of a true pediatric regimen in patients up to the age of 39 years and is currently considered one of several standard-of-care options for AYAs with newlydiagnosed ALL/LBL. ${ }^{13}$ The regimen herein differs from the CALGB 10403 treatment plan in incorporating a twophase induction (with induction II bearing similarities to the consolidation phase of CALGB 10403), HD-MTX versus standard escalating ("Capizzi") MTX, two sequential intensification/re-induction blocks of therapy (vs. one block of interim maintenance and one block of delayed re-induction), 3-years of maintenance chemotherapy for all patients ( $v s$. a shorter 2-year maintenance period for women), a pegaspargase dose of $2,000 \mathrm{IU} / \mathrm{m}^{2}$ versus 2,500 $\mathrm{IU} / \mathrm{m} 2$, and six total doses versus seven total doses of pegaspargase. While it is challenging to compare clinical results directly given differences in trial size, follow-up time, ages of patients enrolled (17-39 years vs. 18-60 years) and period during which patients were enrolled (2007-2012, CALGB 10403 vs. 2014-2017, study herein), the 3-year EFS observed herein (68\% among all patients and $85 \%$ among patients aged $18-39$ years vs. $59 \%$ on CALGB 10403) is encouraging. Long-term follow-up to assess durability of responses observed in this study will further inform interpretation of these results.

Most patients with ALL (83\%) treated on this study exhibited MRD negativity by FACS following induction II. While achievement of MRD negativity following induction I herein did not predict superior long-term DFS and OS among patients with ALL, this study was not powered to perform such a comparison. Other studies have reported a significant difference in DFS/OS among patients achieving MRD negativity by one of several methods at the conclusion of induction phase . $^{13,35}$ The small number of patients with MRD positivity postinduction II does not allow for formal comparison of this group of patients with those who exhibited MRD negativity post-induction II, and alloHCT was favored for patients with MRD positivity post-induction II. It remains unclear whether those patients achieving MRD negativity following two courses (vs. one course) of induction therapy using this approach have inferior longterm disease control or would benefit from earlier introduction of novel agents (e.g., blinatumomab for B-cell ALL), intensified chemotherapy (high-dose cytarabine, nelarabine), or alloHCT in CR1. Inotuzumab ozogamicin and blinatumomab are actively being investigated as 
components of frontline therapy for B-cell ALL/LBL and may facilitate faster achievement of MRD negativity. ${ }^{36,37}$ Incorporating other new agents, such as venetoclax, into this therapeutic backbone may further enhance efficacy and increase rates of MRD negativity post-induction I.

This study has several limitations, foremost being its small size. Samples for central MRD review were available for most, but not all patients, and local MRD was not considered in the primary analysis herein. While standardized criteria for pegaspargase toxicity monitoring and management were provided, institutional practices in this multi-center study likely varied slightly nonetheless. During the conduct of this study, comprehensive genomic profiling of ALL at time of diagnosis, including assessment for targetable alterations characteristic of "Ph-like" ALL, became more common practice. ${ }^{38}$ Such profiling was performed inconsistently in the course of this study, limiting our ability to define the incidence of Ph-like ALL or overall mutational landscape in this cohort. While FACS is a powerful tool for MRD assessment with rapid results, monitoring for the malignant clonal $\mathrm{T}$-cell receptor or immunoglobulin heavy chain re-arrangement by nextgeneration sequencing may have provided even more sensitive detection of MRD. Finally, long-term follow-up remains limited.

In summary, a pediatric-inspired regimen including pegaspargase $2,000 \mathrm{IU} / \mathrm{m}^{2}$ in each of six blocks of intensive therapy, timed to avoid overlapping toxicities, and omitting two myelosuppressive courses of consolidation, resulted in manageable toxicity, high rates of $M R D$ negativity following two-phase induction, and promising long-term efficacy in adults up to aged 60 years with newly-diagnosed ALL/LBL. The addition of active novel agents to such a regimen may increase rates of early $M R D$ negativity and reduce rates of relapse.

\section{Disclosures}

$M B G$ has received research support from Amgen; EKR sits on the advisory board of Agios, Celegne, Genetech, Pfizer and Tolero, has received travel support from Celgene, Novartis and Pfizer, has received research funding from Bristol-Myers Squibb, NS Pharma, Jazz Pharmaceuticals, Novartis, Astellas and Pfizer, consults for Celgene, Novartis, Pfizer and Incyte, is part of the speakers' bureau of Pfizer, Novartis, Incyte and Ariad; AVR is employed by Kite; DD consults for Servier
Pharmaceuticals and Amgen, is part of the speakers' bureau of Servier Pharmaceuticals, Amgen and Adaptive Biotech, and consults for Servier Pharmaceuticals and Amgen; MST sits on the advisory board of Daiichi, Oncolyze, Tetraphase, Jazz Pharmaceuticals, Rigel, KAHR, Abbvie, Nohla, Orsenix, Delta Fly Pharma, BioLineRx and Roche, has received research funding from Cellerant, Biosight, ADC Therapeutics and Abbvie, has patents and royalties at UpToDate, and consults for Daiichi-Sankyo, Oncolyze, Tetraphase, Jazz Pharmaceuticals, Rigel, KAHR, Abbvie, Nohla, Orsenix, Delta Fly Pharma and BioLineRx; JHP has received research funding from Juno Therapeutics and Genentech/Roche, has a consultancy advisory role at Amgen and Juno Therapeutics, and consults for Kite, Incyte, GSK, Autolus, AstraZeneca, Allogene, Novartis, Takeda, Servier and Intellia.

\section{Contributions}

$M B G$ performed the research, including providing care for patients enrolled to the study, analyzed the results, and wrote the paper; EKR, $A V R$ and $P M$ performed the research, including providing care for patients enrolled to the study; $S V, M S$, and $J M S$ gathered and managed the data; $M R$ and $Q G$ performed the research and analyzed the results; JF, MH and SMD analyzed the results; MST, DD and JHP designed and performed the research, including providing care for patients enrolled to the study, analyzed the results, and wrote the paper. All authors critically reviewed the paper.

\section{Acknowledgments}

The authors thank Jessica Wardrope for assistance with organizing and compiling the centralized flow cytometric minimal residual disease analysis reports and thank Dr. Michael Borowitz for providing details of the Johns Hopkins University flow cytometric minimal residual disease analysis methods.

\section{Funding}

Research support was provided by Servier Pharmaceuticals. $M B G$ received funding from Lymphoma Research Foundation, American Society of Hematology, MSK Comedy versus Cancer Grant, Nancy and Jeffrey Heller Giving Fund; JHP received funding from Conquer Cancer Foundation of ASCO, Leukemia and Lymphoma Society Career Development Grant, The Geoffrey Beene Cancer Foundation, National Comprehensive Cancer Center Young Investigator Award, and American Society of Hematology Scholar Junior Faculty Award.

\section{References}

1. Hunger SP, Mullighan CG. Acute lymphoblastic leukemia in children. N Engl J Med. 2015;373(16):1541-1552.

2. Pulte D, Gondos A, Brenner $\mathrm{H}$. Improvement in survival in younger patients with acute lymphoblastic leukemia from the 1980s to the early 21 st century. Blood. 2009;113(7):1408-1411.

3. Rowe JM, Buck G, Burnett AK, et al. Induction therapy for adults with acute lymphoblastic leukemia: results of more than 1500 patients from the international ALL trial: MRC UKALL XII/ECOG E2993. Blood. 2005;106(12):3760-3767.

4. Boissel N, Auclerc MF, Lheritier V, et al. Should adolescents with acute lymphoblastic leukemia be treated as old children or young adults? Comparison of the French FRALLE-93 and LALA-94 trials. J Clin
Oncol. 2003;21(5):774-780

5. Stock W, La M, Sanford B, et al. What determines the outcomes for adolescents and young adults with acute lymphoblastic leukemia treated on cooperative group protocols? A comparison of Children's Cancer Group and Cancer and Leukemia Group B studies. Blood. 2008;112(5):1646-1654.

6. de Bont JM, Holt B, Dekker AW, et al. Significant difference in outcome for adolescents with acute lymphoblastic leukemia treated on pediatric vs adult protocols in the Netherlands. Leukemia. 2004;18(12) 2032-2035.

7. Douer D, Aldoss I, Lunning MA, et al. Pharmacokinetics-based integration of multiple doses of intravenous pegaspargase in a pediatric regimen for adults with newly diagnosed acute lymphoblastic leukemia. J Clin Oncol. 2014;32(9):905-911.

8. Patel B, Kirkwood AA, Dey A, et al. Pegylated-asparaginase during induction therapy for adult acute lymphoblastic leukaemia: toxicity data from the UKALL14 trial. Leukemia. 2017;31(1):5864.

9. Huguet F, Leguay T, Raffoux E, et al Pediatric-inspired therapy in adults with Philadelphia chromosome-negative acute lymphoblastic leukemia: the GRAALL2003 study. J Clin Oncol. 2009;27(6):911918.

10. DeAngelo DJ, Stevenson KE, Dahlberg SE, et al. Long-term outcome of a pediatricinspired regimen used for adults aged 18-50 years with newly diagnosed acute lymphoblastic leukemia. Leukemia. 2015;29(3): 526-534.

11. Huguet F, Chevret S, Leguay $\mathrm{T}$, et al Intensified therapy of acute lymphoblastic leukemia in adults: report of the randomized GRAALL-2005 Clinical Trial. J Clin Oncol. 2018;36(24):2514-2523.

12. Gökbuget N, Beck J, Brandt $K$, et al. 
Significant improvement of outcome In adolescents and young adults (AYAs) aged 15-35 Years with acute lymphoblastic leukemia (ALL) with a pediatric derived adult ALL protocol; results Of 1529 AYAs in 2 consecutive trials of The German Multicenter Study Group For Adult ALL (GMALL). Blood. 2013;122(21):839-839.

13. Stock W, Luger SM, Advani AS, et al. A pediatric regimen for older adolescents and young adults with acute lymphoblastic leukemia: results of CALGB 10403. Blood. 2019;133(14):1548-1559.

14. Earl M. Incidence and management of asparaginase-associated adverse events in patients with acute lymphoblastic leukemia. Clin Adv Hematol Oncol. 2009;7(9):600-606.

15. Amylon MD, Shuster J, Pullen J, et al. Intensive high-dose asparaginase consolidation improves survival for pediatric patients with $\mathrm{T}$ cell acute lymphoblastic leukemia and advanced stage lymphoblastic lymphoma: a Pediatric Oncology Group study. Leukemia. 1999;13(3):335-342.

16. Sallan SE, Hitchcock-Bryan S, Gelber R, et al. Influence of intensive asparaginase in the treatment of childhood non-T-cell acute lymphoblastic leukemia. Cancer Res. 1983;43(11):5601-5607.

17. Stock W, Douer D, DeAngelo DJ, et al. Prevention and management of asparaginase/pegasparaginase-associated toxicities in adults and older adolescents: recommendations of an expert panel. Leuk Lymphoma. 2011;52(12):2237-2253.

18. Asselin BL, Whitin JC, Coppola DJ, et al. Comparative pharmacokinetic studies of three asparaginase preparations. J Clin Oncol. 1993;11(9):1780-1786.

19. Avramis VI, Sencer S, Periclou AP, et al. A randomized comparison of native Escherichia coli asparaginase and polyethylene glycol conjugated asparaginase for treatment of children with newly diagnosed standard-risk acute lymphoblastic leukemia: a Children's Cancer Group study. Blood. 2002;99(6):1986-1994.

20. Muller HJ, Loning L, Horn A, et al. Pegylated asparaginase (Oncaspar) in children with ALL: drug monitoring in reinduction according to the ALL/NHL-BFM 95 protocols. Br J Haematol. 2000;110(2):379-
384.

21.Larsen EC, Devidas $M$, Chen $S$, et al. Dexamethasone and high-dose methotrexate improve outcome for children and young adults with high-risk B-acute lymphoblastic leukemia: a report from Children's Oncology Group Study AALL0232. J Clin Oncol. 2016;34(20):23802388.

22. Wetzler M, Sanford BL, Kurtzberg J, et al. Effective asparagine depletion with pegylated asparaginase results in improved outcomes in adult acute lymphoblastic leukemia: Cancer and Leukemia Group B Study 9511. Blood. 2007;109(10):41644167.

23. Rubenstein JL, Gupta NK, Mannis GN, et al. How I treat CNS lymphomas. Blood. 2013;122(14):2318-2330.

24. Khan RB, Shi W, Thaler HT, et al. Is intrathecal methotrexate necessary in the treatment of primary CNS lymphoma? I Neurooncol. 2002;58(2):175-178.

25. Synold TW, Relling MV, Boyett JM, et al. Blast cell methotrexate-polyglutamate accumulation in vivo differs by lineage, ploidy, and methotrexate dose in acute lymphoblastic leukemia. I Clin Invest. 1994;94(5):1996-2001.

26. Seibel NL, Steinherz PG, Sather HN, et al. Early postinduction intensification therapy improves survival for children and adolescents with high-risk acute lymphoblastic leukemia: a report from the Children's Oncology Group. Blood. 2008;111(5):25482555.

27. Nachman JB, Sather HN, Sensel MG, et al. Augmented post-induction therapy for children with high-risk acute lymphoblastic leukemia and a slow response to initial therapy. N Engl J Med. 1998;338(23):16631671.

28. Christ TN, Stock W, Knoebel RW. Incidence of asparaginase-related hepatotoxicity, pancreatitis, and thrombotic events in adults with acute lymphoblastic leukemia treated with a pediatric-inspired regimen. J Oncol Pharm Pract. 2018;24(4): 299-308.

29. Coustan-Smith E, Mullighan CG, Onciu M, et al. Early T-cell precursor leukaemia: a subtype of very high-risk acute lymphoblastic leukaemia. Lancet Oncol.
2009;10(2):147-156

30. Orvain C, Balsat M, Tavernier E, et al Thromboembolism prophylaxis in adult patients with acute lymphoblastic leukemia treated in the GRAALL-2005 study. Blood. 2020;136(3):328-338.

31. Quist-Paulsen P, Toft N, Heyman M, et al. T-cell acute lymphoblastic leukemia in patients $1-45$ years treated with the pediatric NOPHO ALL2008 protocol. Leukemia. 2020;34(2):347-357.

32. Luskin MR, DeAngelo DJ. T-cell acute lymphoblastic leukemia: Current approach and future directions. Adv Cell Gene Ther. 2019;2(4):e70.

33. Douer D, Yampolsky H, Cohen LJ, et al. Pharmacodynamics and safety of intravenous pegaspargase during remission induction in adults aged 55 years or younger with newly diagnosed acute lymphoblastic leukemia. Blood. 2007;109(7): 2744-2750.

34. Lanvers-Kaminsky C, Niemann A, Eveslage $\mathrm{M}$, et al. Asparaginase activities during intensified treatment with pegylated E. coli asparaginase in adults with newly-diagnosed acute lymphoblastic leukemia. Leuk Lymphoma. 2020;61(1):138-145.

35. Patel B, Rai L, Buck G, et al. Minimal residual disease is a significant predictor of treatment failure in non T-lineage adult acute lymphoblastic leukaemia: final results of the international trial UKALL XII/ECOG2993. Br J Haematol. 2010;148 (1):80-89.

36. Inotuzumab Ozogamicin and Blinatumomab in treating patients with newly diagnosed, recurrent, or refractory CD22-positive B-lineage acute lymphoblastic leukemia. [cited; available from: https://clinicaltrials.gov/ct2/show/NCT037 39814

37. Combination chemotherapy with or with out blinatumomab in treating patients with newly diagnosed BCR-ABL-negative $B$ lineage acute lymphoblastic leukemia. https://clinicaltrials.gov/ct2/show/NCT0 2003222

38. Roberts KG, Li Y, Payne-Turner D, et al. Targetable kinase-activating lesions in $\mathrm{Ph}$ like acute lymphoblastic leukemia. N Engl Med. 2014;371(11):1005-1015. 